\title{
Optimizing Materials Properties using Modeling Tools to Simulate Solidification and Heat Treatment
}

\author{
Fan Zhang
}

Computer aided materials design has become increasingly important in modern materials design and development. Many modeling tools have been developed or are currently under development within the scope of Integrated Computational Materials Engineering (ICME). These modeling tools are used to understand the effects of alloy chemistry and processing conditions on the evolution of microstructure and ultimately mechanical properties, so that the materials performance can be "designed" and "optimized" even before the materials are produced. The following three papers demonstrate such efforts for the simulation of three different processes: solidification, homogenization, and precipitation.

The paper titled "Modeling of Alloy Casting Solidification" by J. Guo and M. Samonds presents casting simulation of the solidification process. As stated in the paper: "Alloy casting solidification processes involve many physical phenomena such as chemistry variation, phase transformation, heat transfer, fluid flow, microstructure evolution, and mechanical stress." Two commonly used approaches for microscopic modeling of solidification are reviewed; the deterministic method and the stochastic method. The deterministic method is used for the simulation of phase fraction, dendrite grain size, and the secondary dendrite arm spaces. The as-cast room temperature yield strength can be calculated based on the microstructure predicted. The cellular automaton (CA) model based on the stochastic method can be used to simulate the dendritic structure at different solidification stages. Defects formed during the casting process, such as porosity, hot tearing, and macrosegregation were then discussed in the paper. The formation of these defects is found to be related to thermodynamics, fluid flow, thermal, and/or stress, and all these factors are intertwined.

Next, “An Integrated Computational Tool for Precipitation Simulation" by Weisheng Cao et al. presents an integrated computational thermodynamics and kinetics approach to simulate the microstructural evolution and the corresponding mechanical properties during the precipitation process. Three built-in precipitation models for microstructure simulation at multi-levels were discussed in the paper, which are the JMAK model (Johnson-MehlAvrami-Kolmogorov) for the estimation of the overall transformation rate, the fast-acting model based on the Langer and Schwartz theory for the simulation of the evolution of particle number density and mean size, and the more advanced KWN (Kampmann \& Wagner Numerical) model for the predication of the full evolution of particle size distribution (PSD) in addition to average quantities. The modeling tools were applied to study a model $\mathrm{Cu}$ based alloy and a number of Ni-based superalloys to demonstrate its capability in microstructural simulation. This paper also discusses the integration of the microstructure information with strengthening models for the prediction of the age hardening behavior. Aluminum alloys (AA6XXX series) are used to demonstrate the application in this regard.

The paper titled "Microstructural Modeling of the Homogenization Heat Treatment for AA3XXX Alloys" by Qiang Du et al. mainly discusses the microstructure evolution during homogenization of AA3XXX alloys. The model developed by the authors is fully coupled with thermodynamic calculation, and is able to simulate the reduction of the microsegregation formed during solidification, the nucleation, growth and coarsening of intra-granular dispersoids, and the growth/dissolution of inter-granular constituent particles during the homogenization process of AA3XXX alloys. Simulation was performed for an AA3003 alloy to predict the microstructure change under different homogenization heat treatment conditions. With the positive experimental validations of the simulation results the authors concluded that the model has a potential to be used as a valuable tool for optimizing the heat treatment parameters of the AA3XXX alloys.

Fan Zhang is the president of CompuTherm, LLC in Madison, Wisconsin and is the advisor to JOM from the Alloy Phases Committee of the Electronic, Magnetic \& Photonic Materials Division of TMS

\section{Fan Zhang is a TMS Member!}

To read more about her, turn to page 8. To join TMS, visit www.tms.org/Society/Membership.aspx. 